\title{
NIETZSCHE, MACH Y LA METAFÍSICA DEL YO ${ }^{1}$ \\ Nietzsche, Mach and the Metaphysics of Self
}

\author{
Pietro Gori
}

Resumen: En un importante aforismo de Más allá del bien y del mal, Nietzsche declara que cualquiera que quiera admitir el principio cartesiano del «yo pienso», se encuentra expuesto a una serie de "problemas de la metafísica», el primero de ellos el relativo a la existencia de un yo y a su valor de causa de los actos del pensamiento. Con el presente artículo se quiere ofrecer un examen de esta posición - en muchos aspectos conforme a las tesis del científico austriaco Ernst Mach - con el objetivo de individuar los rasgos característicos de la noción de yo definida por Nietzsche y, por tanto, los fundamentos de su teoría de la mente.

Palabras clave: yo - pensamiento - metafísica - psicología

ABSTRACT: In an important aphorism of Beyond Good and Evil Nietzsche writes that anyone who believes in «immediate certainties» such as «I think» encounters a series of «metaphysical questions». The most important of these «problems of intellectual knowledge» is the existence of an ' $I$ ', as much as our believing it to be the cause of thinking. This article will deal with this observation - that in many ways is in compliance with the ideas of the Austrian scientist Ernst Mach - and will show how Nietzsche describes the «ego» and, therefore, which are the grounds of his view on the mind-body problem.

Keywords: I - Thought - Metaphysics - Psychology

En el aforismo 16 de Más allá del bien y del mal, Nietzsche presenta algunas observaciones relativas al pensamiento, concentrándose en particular en la noción de yo, que viene comúnmente identificada como elemento del origen del proceso intelectivo. Nietzsche expone la cuestión en estos términos, analizando cuáles son las cosas que se asumen y sobre las que sería necesario rendir cuentas antes de poder hablar de un «yo pienso", sobre todo si a esto se le intenta atribuir el valor de certeza inmediata ${ }^{2}$ :

Cuando analizo el proceso que se expresa en la proposición «yo pienso», obtengo una serie de afirmaciones temerarias cuya justificación resulta difícil, y quizás impo-

1. Título del original: «Nietzsche, Mach e la metafisica dell'io».

2. P. Bornedal ha dedicado un capítulo a la crítica nietzscheana y kantiana del «yo pienso» de Descartes en su libro The Surface and the Abyss, Berlin: De Gruyter, 2010, cap. 3. Cf. también N. Loukidelis, «Quellen von Nietzsches Verständnis und Kritik des cartesischen cogito, ergo sum»: Nietzsche-Studien 34 (2005), 300-309. 
sible, - como, por ejemplo, que yo soy el que piensa, que tiene que existir algo en general que piensa, que pensar es una actividad y el efecto de un ser que es pensado como causa, que existe un «yo»y, finalmente, que está establecido qué es lo que hay que designar con la palabra pensar, - que yo sé qué es pensar.

Unas líneas más adelante, se acota la cuestión, concentrando su atención sobre la determinación de la noción de yo, la cual juega un papel fundamental en la representación común, inmediata, no-filosófica de los actos del pensamiento:

En lugar de aquella «certeza inmediata» en la que el pueblo, dado el caso, puede creer, el filósofo encuentra entre sus manos una serie de problemas de la metafísica, verdaderas cuestiones de conciencia del intelecto, que se formulan así: «¿De dónde saco el concepto de pensar? ¿Por qué creo en la causa y en el efecto? ¿Qué me da a mí derecho a hablar de un yo e incluso de un yo como causa, y, finalmente, incluso de un yo como causa de los pensamientos?».

El orden de problemas que Nietzsche plantea parece ser bastante claro, así como el hecho de que él tienda a una subdivisión inmediata de la noción de «yo pienso» en dos elementos que la componen: el acto del pensamiento, cuya determinación no tiene nada de clara e inmediata, y el presunto sujeto de este acto, sobre cuyo origen y caracterización Nietzsche se pregunta de modo especial. Los dos elementos en cuestión son obviamente correlativos, perteneciendo al mismo horizonte discursivo. Sin embargo, el modo en que vienen comúnmente descritos da vida a un claro dualismo, donde ciertamente el acto que se desarrolla y el objeto que subyace a su desarrollo son en algún modo distintos, incluso sólo desde el punto de vista lógico. Objeto del presente artículo será el segundo de los elementos en cuestión, del que tengo intención de discutir el valor sustancialísta y (por lo tanto) metafísico, tratando luego de sacar algunas conclusiones sobre el modo en que Nietzsche entiende la cuestión. En la práctica, mi objetivo es desarrollar el último de los interrogantes que él plantea en el aforismo en cuestión: ¿Qué es lo que me da derecho a hablar de un yo como causa de los pensamientos?

\section{ERNST MACH: EL YO COMO «UNIDAD IDEAL»}

Para afrontar este tema y valorar la solución que da Nietzsche haré referencia a la contribución que hizo en aquellos mismos años el científico austríaco Ernst Mach, el cual trabajó sobre la cuestión psicológica del yo, analizándola a partir del plano fisiológico de formación de los procesos de pensamiento y presentó una concepción monística a partir de la cual se puede intentar dar una solución al problema de la relación cuerpo-mente ${ }^{3}$. La elección de esta referencia no es obviamente casual, desde el momento en que un ejemplar de Análisis de las sensaciones, publicado por Mach en 1886, se encuentra en la biblioteca de

3. La utilización de Mach como autor de referencia para discutir algunas posiciones de Nietzsche - de modo particular las relativas al sensualismo declarado en JGB $\$ 15$ - es la línea seguida por N. Hussain en su «Reading Nietzsche through Ernst Mach», en T. Brobjer y G. Moore (eds.), Nietzsche and Science, Aldershot: Ashgate, 2004, pp. 111-129. 
Nietzsche ${ }^{4}$. Mach es, por tanto, un autor conocido por Nietzsche y con el que es posible confrontar sus posiciones, en la medida en que un estudio en profundidad nos lleve a concluir que él no puede contarse entre las fuentes directas de su pensamiento. Aunque, de hecho, algunas posiciones de Nietzsche en materia epistemológica sean parecidas a las ideas de Mach, el estudio fisiológico de sus escritos y, en particular, la cronología de las referencias textuales no permiten convalidar el influjo directo. Personalmente, más bien que sostener - como se ha hecho recientemente- que Nietzsche puede haber acogido algunas posiciones de Mach, que seguramente circulaban a partir de 1872, considero preferible admitir que los dos autores hayan hecho referencia a fuentes comunes, elaborando luego de manera personal, según los respectivos campos de investigación, las concepciones epistemológicas discutidas en el debate de la época ${ }^{5}$. La referencia a Mach permite ampliar el campo de observación a temas discutidos en la segunda mitad del siglo XIX en el ámbito científico, lugar en que fue debatida de manera diversa la cuestión relativa a la determinación en términos fisiológicos de la problemática noción de «alma» ${ }^{6}$. Por lo que ha sido posible revelar a partir del estudio de las fuentes de su pensamiento, puede afirmarse que Nietzsche demostró un vivo interés por esta temática y estaba informado sobre las publicaciones relativas a ella, asimilando algunas de las posiciones de mayor relieve para luego orientarlas en un sentido exquisitamente filosófico. Sus observaciones sobre la cuestión de la subjetividad y del pensamiento, por lo tanto, se analizan teniendo en cuenta esta referencia histórica y cultural, sin la cual tales observaciones estarían privadas de un fundamento ${ }^{7}$. Mach, por su parte, juega un papel particularmente significativo, compartiendo la línea de investigación puesta en marcha por Fechner y Helmholtz para intervenir de manera determinante no tanto en la configuración de una psicología fundada sobre bases fisiológicas, sino más bien en la determinación de un ámbito de referencia común a partir del cual sea posible una reconsideración de estas dos disciplinas que las libere del bagaje metafísico sobre el que han sido edificadas.

El objetivo declarado de Análisis de las sensaciones es poner de relieve cómo la distinción entre el plano psíquico y el plano físico es de hecho puramente lógica y no puede ser sostenida en el sentido de un verdadero y auténtico dualismo entre los ámbitos (aparentemente) distintos de la física y de la psicología. Mach, de hecho, rechaza la referencia a una $\cos a$ en $s i^{8}$ cualquiera y sostiene que el plano de la realidad desde el que hay que moverse es el de los elementos (cuyo valor es asumido en sentido exclusivamente metodológico) ${ }^{9}$. Por lo

4. Cf. G. Campioni et al. (ed.), Nietzsches persönliche Bibliothek, Berlin: De Gruyter, 2002, p. 382. El texto de Mach en su primera edición llevaba el título Beiträge zur Analyse der Empfindungen.

5. Para un tratamiento en profundidad de tales cuestiones me remito a mi Il meccanicismo metafisico. Scienza, filosofia e storia in Nietzsche e Mach, Bologna: Il Mulino, 2009.

6. Cf. M. Ash y W. Woodward (eds.), The Problematic Science. Psychology in the Nineteenth Century Thought, New York: Praeger, 1982, y R. Martinelli, Filosofia e psicofisica da Kant a Carnap, Macerata: Quodlibet, 1999.

7. Cf. A. Orsucci, Dalla biologia cellulare alle scienze dello spirito, Bologna: Il Mulino, 1992.

8. Cf. E. Mach, Die Analyse der Empfindungen und das Verhältnis des Physischen zum Psychischen, Ernst Mach Studienausgabe, vol. 1, Berlin: Xenomoi, 2008 (citaremos como AE), I, 13, p. 35n.

9. La determinación de los «elementos» de la que habla Mach es quizás uno de los aspectos más delicados de su pensamiento. Sobre esto véase E. Banks, Ernst Mach's World Elements: a Study in Natural Philosophy, Dodrecht/Boston: Kluwer, 2003. 
tanto, «el principio del paralelismo completo entre ámbito psíquico y físico» ${ }^{10}$, que guía su investigación, tiene la particularidad de no hacer referencia a una entidad absoluta, identificando más bien un sustrato que adquiere características peculiares según la perspectiva desde la que se lo examina. Las sensaciones identificadas por el monismo neutral de Mach están, en efecto, privadas de ninguna determinación cualitativa, la cual emerge solamente a continuación de la relación instituida con las entidades corpóreas que las registran. Por este motivo Mach puede insistir en no admitir una diferencia sustancial entre los dos ámbitos, encontrando casi obvia esta asunción como consecuencia de una premisa general por la que a cada fenómeno psíquico le corresponde un fenómeno físico y viceversa. Dicho sintéticamente: «Los elementos encontrados por nosotros en la experiencia, cuya conexión indagamos, son siempre los mismos, de un solo tipo y sólo en función de su conexión se nos presentan ora como elementos físicos ora como elementos psíquicos» ${ }^{11}$.

La determinación cualitativa de los elementos depende por lo tanto de la relación dentro de la cual se encuentran cada vez inmersos y cada componente fundamental puede entrar de nuevo ya sea en un complejo identificable en los términos de una entidad «física», ya sea en uno definible sobre el plano "psíquico». La dimensión de la sustancia pierde por lo tanto su propia consistencia ontológica, mientras que el componente funcional adquiere un relieve; en otras palabras, no hay fenómenos físicos o psíquicos, sino solamente una interpretación física o psíquica de los mismos acontecimientos, y hablar de cuerpo o de yo significa hacer referencia al mismo plano, que es simplemente observado desde perspectivas diferentes. En definitiva, en ambos casos se está dando un nombre a complejos de elementos que se distinguen de los otros por el mero hecho de poseer una persistencia relativamente mayor, y es precisamente esta relativa permanencia lo que constituye el principal elemento de dificultad para el sujeto cognoscente, en cuanto que la tendencia es la de absolutizar su duración haciendo de una realidad en devenir el reino metafísico de la entidad sustancial.

A la luz de estas observaciones es posible afrontar la cuestión específica del yo en Mach. Él lo define en primer lugar como un conjunto de sensaciones que se refieren a una dimensión corporal individual, privándolo así de cualquier determinación que prescinda de aquel complejo de disposiciones y sentimientos:

Como relativamente persistente se presenta como un conjunto de recuerdos, disposiciones, sentimientos, ligado a un determinado cuerpo que viene designado como «yo». Yo puedo ocuparme de esta o aquella cosa, puedo estar tranquilo y alegre o también irritado y de mal humor. Queda son embargo (prescindiendo de los casos patológicos) una suma de elementos persistentes $\underline{\text { itales de consentir? }}$ el reconocimiento de la identidad del yo. Por otra parte, también el yo tiene una persistencia sólo relativa. La aparente persistencia del yo consiste sobre todo en la continuidad, en la lentitud de la mutación ${ }^{12}$.

10. AE IV, 3, p. 66.

11. Ibid., p. 67.

12. AE I, 2, p. 13 
Según esta perspectiva, el yo no es nada comparado con la multiplicidad de elementos que el cuerpo registra, y por tanto su origen es puramente lógico y se deriva de una exigencia de unidad que finaliza con el reconocimiento; a través de la determinación de un alma — porque es de esto de lo que se trata- es de hecho posible identificar a una persona y seguir sus cambios, admitiendo su identidad sustancial. La necesidad de orientación mueve al intelecto a construir una referencia unitaria que pueda ser utilizada para dar un nombre al contenido en mayor medida persistente de un conjunto de sensaciones.

El razonamiento que Mach desarrolla en su escrito de 1886 conduce a una disgregación de la noción de yo, que con él pierde todo el valor metafísico que tradicionalmente se le podía atribuir. El objeto que funda el análisis psicológico no posee una esencia diferente de cualquier otra entidad presente en el mundo «real», desde el momento en que deriva del mismo sustrato del que están formados los cuerpos. No sólo eso: la absoluta neutralidad de los elementos, que el científico elige asumir como referencia antes de la descripción del mundo, permite abandonar cualquier tipo de determinación ontológica sobre la que se funda la tradicional distinción entre materia y anima. Cuerpos y yo son simplemente construcciones del pensamiento, el cual registra diversas modalidades de relación entre los elementos clasificándolos en uno de los dos ámbitos, sin que con esto haga referencia a una manifestación cualitativa de ellos.

$\mathrm{El}$ análisis que Mach hace del yo se fija particularmente en hacer evidente el primado ontológico de los elementos respecto al complejo unitario puramente nominal de las nociones elaboradas por el intelecto. El concepto psicológico fundamental viene a ser definido por tanto a partir de la formación de una «unidad ideal», que reúne «todos los elementos que están más estrechamente relacionados con el dolor y el placer», y que «consiente la economía del pensamiento [...] La delimitación del yo se produce por eso instintivamente, llega a ser habitual y se consolida quizás sin ninguna duda a través de la transmisión hereditaria» ${ }^{13}$. Sobre el plano estrictamente ontológico, es propiamente la dependencia completa del yo de los elementos (reconocidos en su relación con la corporeidad individual como sentimientos, reflejos, y, más en general, sensaciones) la que contribuye a la eliminación de su valor metafísico. Son estos, de hecho, los que representan el «material» que, una vez conexo, viene a constituir el alma individual, y sin el cual no habría nada que circunscribir:

El yo no es el hecho primario sino los elementos (sensaciones). [...] Los elementos forman el yo. "Yo percibo el verde» significa que el elemento verde aparece en un cierto complejo de elementos (sensaciones, recuerdos). Cuando dejo de percibir el verde, cuando muero, los elementos ya no se vuelven a presentar

13. AE I, 12, p. 29. Afrikan Spir, en su Forschung nach der Gewissheit in der Erkenntnis der Wirklichkeit (Leipzig, 1869) ha mantenido una posición muy similar a esta al describir la noción de yo (se pueden ver sobre todo las pp. 220-223). Como ya se ha demostrado ampliamente, Spir, de manera distinta que Mach, ha sido una fuente directa de Nietzsche, del que ha leido en su juventud Denken und Wirklichkeit, Versuch einer Erneuerung der kritischen Philosophie, del 1873 (cf. M. S. Green, Nietzsche and the Transcendental Tradition, Chicago: University of Illinois Press, 2002, y P. D'iorio, «La superstition des philosophes critiques. Nietzsche et African Spir»: NietzscheStudien 22 [1993], 257-294). Spir constituye evidentemente una de las referencias comunes a los dos autores, así como el medio para la asimilación por parte de Nietzsche de algunas posiciones que desarrollaron la filosofía crítica de Kant. 
en la asociación corriente a la que yo estaba acostumbrado. Con esto está dicho todo. Ha dejado de existir solamente una unidad ideal, creada en vistas a una economía del pensamiento, no una unidad real ${ }^{14}$.

Aquello que cuenta para poder fundar una psicología es que haya continuidad

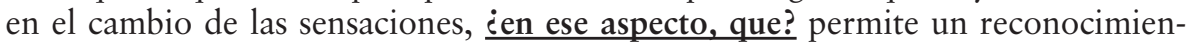

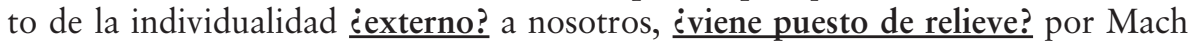
como esencial para el mismo sujeto que reflexiona sobre sí mismo: él, de hecho, escribe que sin la "cadena de recuerdos», que se encuentran ligados en una línea de continuidad, «no podría considerar éotra cosa que el muchacho que era? ${ }^{15}$. Tal continuidad es una vez más algo ficticio, inventado por el pensamiento para dar cohesión a la subjetividad individual, la cual necesita de una determinación tanto espacial como temporal. La unidad del yo tiene que poder valer ya sea como principio de determinación de los otros sujetos, ya sea como núcleo estable que permanece en la base del incesante cambio que ¿̇contradistingue? la actividad espiritual: «La continuidad es por lo tanto solamente un medio para preparar y asegurar el contenido del yo. Este contenido, y no el yo, es lo principal» ${ }^{16}$. La conclusión de Mach es que resulta imposible salvar al yo, en el sentido de que, siguiendo la perspectiva abierta por su monismo, viene a perderse en la conexión de los elementos y es, por tanto, necesario abandonar cualquier pretensión de atribuirle una existencia autónoma. En otras palabras, una vez admitido que el sujeto se compone de sensaciones, es imposible tratar de mantener íntegra la presunta unidad psíquica, tal y como lo ha hecho en el pasado la ciencia; esta última, movida por la costumbre de «tratar el complejo no analizado del yo como una unidad indivisible», ha intentado ante todo aislar del cuerpo «el sistema nervioso, como sede de las sensaciones», por lo tanto el cerebro, hasta admitir la existencia de un simple punto como sede del alma, que no está obviamente en condiciones de encontrar ${ }^{17}$.

Incluso sosteniendo una crítica radical a la investigación científica de su tiempo, Mach se da cuenta, sin embargo, de la necesidad de distinguir dos planos del discurso, salvaguardando el valor del yo como noción de referencia que no es posible abandonar sobre el plano meramente práctico. Su observación conclusiva, contenida en un capítulo añadido a la segunda edición del texto, es que «no obstante todo esto, para mi concepción instintiva el yo es la cosa más importante y más persistente. Es aquello que une en conjunto todas mis experiencias y la fuente de toda mi actividad $»^{18}$. Antes de confrontar estas posiciones con las ideas de Nietzsche sólo hay un aspecto que hemos de considerar. En la continuación del párrafo en el que Mach contesta la andadura de las investigaciones científicas sobre el tema del yo, de hecho, él cita un pasaje de Lichtenberg:

Afirma Lichtenberg en sus observaciones filosóficas: «Llegamos a ser conscientes de ciertas representaciones que no dependen de nosotros; otras, por el contrario, al menos lo creemos, dependen de nosotros; ¿dónde está la línea de demarcación? Conocemos solamente la existencia de nuestras sensaciones, representaciones y pensamientos. El pensar debería ser considerado de una manera

\footnotetext{
14. Ibid., pp. 29-30.

15. AE I, 2, p. 13

16. AE I, 12, p. 30.

17. Ibid., p. 32.

18. AE XV, 2, p. 325
} 
igualmente impersonal que el relampaguear (debería decirse «se piensa», como se dice «relampaguea»). Decir «cogito» es ya demasiado cuando se lo traduce con un «yo pienso». Suponer el yo, postularlo, es una necesidad práctica ${ }^{19}$.

Ahora bien, es suficientemente conocido el hecho de que este pasaje es la referencia implícita detectable que subyace al aforismo 17 de Más allá del bien y del mal, lugar en el que Nietzsche continúa su crítica a la noción cartesiana del ego cogito, observando la necesidad de hacer referencia a un sujeto impersonal, o sea, a la misma acción del pensamiento, sin tener que inventar a la fuerza un sujeto que subyace a este acontecimiento ${ }^{20}$. Por tanto, parece ser que Lichtenberg es una fuente común de reflexión de los dos autores, y este pasaje suyo en particular constituye el punto de contacto entre las observaciones psicológicas de Mach y las páginas del texto de Nietzsche, al que será oportuno hacer referencia para examinar el modo en que este último haya definido la noción de yo.

\section{SUJETO Y ACCIÓN}

La idea que Nietzsche expone al tratar la noción del yo es ¿̇diversamente? asimilable a las posiciones del científico austriaco, ¿condividiendo? sobre todo la orientación crítica con que Mach afronta la cuestión del saber metafísico. Este se concentra de modo particular en el carácter puramente ficticio que ¿contradistingue? la unidad psicológica fundamental, poniendo el acento sobre los rasgos generales de la actividad del pensamiento, descrita por él en términos puramente fisiológicos. El yo, en particular, es considerado como el producto de una actividad secundaria del pensamiento, la actividad lógica, la cual interviene en la atribución de un sujeto en el interior de un proceso que resulta constitutivamente privado de él. En vez de ser el agente de una acción que se desarrolla de una manera absolutamente no intencional, según Nietzsche, el yo es un simple producto del desarrollo del pensamiento:

Lo que me separa más profundamente de los metafísicos es esto: yo no acepto que sea el «yo» lo que piensa: antes bien, considero el yo mismo como una construcción del pensamiento, del mismo rango que «materia», «cosa», «sustancia», «individuo», «fin», «número»; por tanto sólo como ficción regulativa, con cuya ayuda se introduce, se inventa, en un mundo del devenir, una especie de estabilidad y por tanto de "cognoscibilidad». [...] El pensamiento es el que pone el «yo»: pero hasta ahora se creía, como cree el «pueblo», que en el «yo pienso» se encuentra algo inmediatamente cierto y que en este «yo» está dada la causa del pensamiento ${ }^{21}$.

El «yo» es un producto del pensamiento, no es más que una entidad conceptual cuyo valor se limita a su utilidad práctica con el fin de categorizar el mundo

19. AE I, 12, p. 34

20. Nikolaos Loukidelis ha dedicado su investigación doctoral a un examen de este importante aforismo, concentrándose sobre todo en la referencia a Lichtenberg. Su tesis, defendida en 2010 en la Humboldt-Universität de Berlín, tiene como título: Es denkt. Ein Kommentar zum Aphorismus 17 von Jenseits von Gut und Böse.

21. FP III 781, 35 [35]. 
y su orden puramente lógico 22 . Este se coloca sobre el mismo plano que todos aquellos elementos sustanciales a los que se suele atribuir una existencia absoluta y cuya génesis se debe a una operación de traducción del mundo exterior en un lenguaje que pueda ser comprendido y utilizado por nuestro entendimiento -en aquella perspectiva de producción de formas que según Nietzsche caracteriza la modalidad cognoscitiva del hombre- - Sosteniendo un punto de vista que, como se puede ver fácilmente, presenta muchos elementos en común con la posición de Mach, Nietzsche reconoce que el yo puede desempeñar el mismo papel que el desempeñado por los átomos y por cualquier entidad sustancial, proporcionando un punto de referencia estable para fijar el mundo del devenir y hacerlo calculable. En general, la operación puramente intelectiva que lleva a la determinación de una unidad individual respeta las modalidades de aquella tendencia a admitir un ser en una realidad caracterizada por el cambio de las formas, y por lo tanto permanece dentro de la perspectiva del pensamiento que funda todo saber metafísico ${ }^{23}$.

La reflexión de Nietzsche, sin embargo, no se detiene en este punto. El yo, de hecho, posee una característica que lo distingue de las otras entidades sustanciales, cuyo origen es el resultado de una operación de simplificación de una multiplicidad caótica a través del aislamiento de formas fijas y unitarias. Para dar unidad a los sentimientos, a las percepciones, a los recuerdos, se busca de hecho algo activo, que sea capaz de actuar como origen de tales disposiciones: como su causa. La unificación de la multiplicidad de las sensaciones se lleva a cabo a través de la identificación de una entidad espiritual, circunscribible sólo a partir de su capacidad de acción; también por este motivo es absurdo querer atribuirle un valor de existencia, como si fuese posible indicar y describir aquello de donde emana una acción, cuando lo único que es posible detectar son los efectos de la acción misma. En la interpretación de Mach, por lo tanto, el sujeto no es otra cosa que una creación de la actividad representativa, una errónea simplificación generada con la que se cree poder «designar como tal la fuerza que pone, inventa, piensa, a diferencia del poner, inventar, pensar mismo singular en cada caso» ${ }^{24}$.

22. Se suele decir, para ponderar el carácter puramente teórico de estas reflexiones, que en Nietzsche el valor del yo tiene un espacio relevante también en un discurso que se desarrolle sobre el plano ético. Con estas observaciones, Nietzsche no parece querer eliminar la noción de individualidad; más bien actúa en dirección de una reconfiguración suya, cambiando su dimensión ontológica y preguntándose sobre qué le ocurre al hombre en el momento en que esta «nueva visión» es asimilada. La intención crítica de Nietzsche no es por tanto puramente destructiva y, más aún, su filosofía surge a partir de este tipo de reconsideración de algunas «verdades» adquiridas, funcionando en el sentido de un esclarecimiento iluminista: una operación que parece ser compatible de modo particular con los objetivos de la filosofía científica que se desarrolla en torno a la figura de Mach (cf. P. Frank, Modern Science and its Philosophy, Cambridge: Harvard UP, 1946).

23. En un cuaderno de 1887-1888, Nietzsche señala de una manera todavía más explícita esta dependencia de la lógica de la noción puramente ficticia del yo, evidenciando en particular la necesidad de su admisión a fin de que el hombre se imponga teóricamente sobre el mundo. En el fragmento 9[89], sobre todo, habla del «yo» como de «un ente (no afectado por el devenir)», confirmando el hecho de que el reconocimiento de una subjetividad individual no corresponde a la descripción de un contenido que es posible aislar realmente, pero lleva más simplemente a circunscribir un núcleo de permanencia con el que se pueda relacionar las sensaciones y las modificaciones que pueden adscribirse a la esfera del sentido interno.

24. FP III 123, 2[152]. La voluntad de identificar un sujeto agente que subyacería al desarrollo de los acontecimientos es un tema sobre el cual Nietzsche ha insistido mucho, lamentando sobre todo la tendencia del hombre a antropomorfizar la dinámica natural. Esto es evidente, por ejemplo, 
La perspectiva general dentro de la cual se mueve es por tanto la que se expresa sistemáticamente en la conclusión que establece Nietzsche en la Genealogía de la moral, según la cual «no existe ningún 'ser' por debajo del hacer, del obrar, del devenir; 'aquel que hace' no está más que ficticiamente unido al obrar - el obrar lo es todo» ${ }^{25}$. Rechazando esta observación en el caso específico del pensamiento, es fácil ver cómo es dable encontrar en ella una posible repuesta a la cuestión de la relación cuerpo-mente. El resultado de las reflexiones de Nietzsche conduce de hecho a una perspectiva según la cual el pensamiento no se distingue de la actividad fisiológica que lo determina, y por tanto no hay ningún dualismo sujeto-objeto que lo sustancie. No existe un agente de los pensamientos; estos se producen necesariamente a partir del proceso interno del organismo. Del mismo modo, no hay ningún sujeto distinto de las sensaciones generadas por nuestra facultad perceptiva; ellas se nos manifiestan de una manera espontánea y solo después son registradas a nivel consciente, y de este modo son organizadas y «comprendidas». Es en este momento cuando interviene la noción de yo, una contribución de la lógica para categorizar las sensaciones (que son así relacionadas con un sustrato unitario), cuya utilidad es tan indudable como lo es su inconsistencia ontológica.

Pero vayamos por partes y veamos dónde y en qué términos Nietzsche expone sus propias reflexiones al respecto. El tratamiento más explícito de la concepción fisiológica del pensamiento se contiene en un par de fragmentos de 1884-1885, cuyo grado de elaboración lleva a imaginar que Nietzsche tuviese en mente preparar una versión para la imprenta, probablemente para añadirla

en el caso de la interpretación del vínculo entre causa y efecto, modelo de una dinámica puramente necesaria que es comúnmente descrita en términos del obrar humano, absolutamente intencional. La tendencia es la de proyectar en las cosas el modelo de la actividad a la que estamos habituados, atribuyendo los caracteres propios del sujeto a la fuerza que mueve la realidad material. (Cf. FP IV 101, 2[83] y FP IV 542, 14[95].) Este aspecto había sido ya puesto de relieve por Lange en las páginas de su Historia del materialismo, cuando afirmaba que la fuerza viene «personificada simultáneamente» con la idea de materia, «dado que nos la figuramos como una emanación y, por decirlo así, como un instrumento de la materia [...] Aquello que en la idea de fuerza es antropomórfico pertenece todavía a la idea de materia, ¿̇sobre la cual, como sobre cada sujeto, hace referencia porción del propio yo?" (F. A. Lange, Die Geschichte des Materialismus, Frankfurt a. M. 1974 [1882], vol. 2, p. 651). Una observación que puede confrontarse directamente con el siguiente apunte: "Causa' y 'efecto': revisado psicológicamente, es la creencia que se expresa en el verbum, activum y passivum, hacer y padecer. Es decir: la división del acontecer en un hacer y un padecer, la suposición de un agente es previa. Detrás está la creencia en el agente: como si, una vez sustraída toda acción del 'agente', este aún permaneciera. Lo que aquí siempre ejerce de guía es la 'representación del yo': todo acontecer es interpretado como hacer: con la mitología de un ser correspondiente al 'yo'» (FP IV 188, 1886, 7[1]). Considerando la tendencia de atribuir a la realidad las propias cualidades personales, debida principalmente al necesario perspectivismo que impide al hombre una observación objetiva y desvinculada de su limitado punto de vista, Nietzsche llega a admitir que la idea de «sujeto» está en la base de la misma categorización de la realidad, en el sentido de que aquello sería el modelo que lleva a la determinación de «seres» y «cosas» entendidas como elementos unitarios. El sujeto es en la práctica aquella referencia que permite reconocer un ente mientras atraviesa sus estados de desarrollo, es el núcleo que garantiza la determinación de una identidad de las cosas. Sin la referencia a este elemento puramente psicológico faltarían los presupuestos para tematizar la misma noción de sustancia; es de hecho el apego a la idea de un sustrato unitario que pueda reunir nuestros múltiples estados lo que nos impulsa a admitir que haya una unidad de fondo, un elemento fijo e inmutable que pueda decirse «real» y que desempeñe el papel de la base para edificar el mundo del devenir (FP IV 303, 10 [19]).

25. GM I $\mathbb{1 3}$. 
a Más allá del bien y del mal. El primero de los apuntes conserva también un esbozo del título: Aquello que es involuntario en el pensamiento. En él se lee:

El pensamiento emerge a menudo mezclado y oscurecido por una aglomeración de pensamientos. Nosotros lo sacamos, lo limpiamos, lo ponemos sobre sus pies y vemos cómo camina - iTodo esto muy deprisa! [...] El origen del pensamiento se nos oculta; es muy probable que sea síntoma de una situación más amplia, como todo sentimiento - : en que justamente es él el que viene y ningún otro, que justamente viene con esta mayor o menor claridad [...], en todo eso se expresa mediante signos algo de una situación compleja ${ }^{26}$.

De lo que Nietzsche está hablando es evidentemente de una forma de pensamiento consciente, que es considerado por él como el simple signo de una actividad que se desarrolla a un nivel "prepsicológico» ${ }^{27}$. Cuando se habla de nivel psicológico se hace referencia, por el contrario, a lo que emerge de un proceso mucho más vasto, que, sin embargo, se verifica en profundidad, a nivel orgánico, y obviamente de manera absolutamente privada de intencionalidad. El origen del pensamiento se nos manifiesta como algo desconocido, no manifestándose a nivel consciente, mientras que todo aquello de lo que somos conscientes no son más que los efectos de la articulación pulsional que constituye la dinámica del organismo. Este discurso se retoma y se profundiza en el segundo apunte, escrito en 1885:

El pensamiento, en la forma en que llega, es un signo equívoco que necesita de interpretación, más exactamente, de una restricción y delimitación voluntarias, hasta que por fin resulta unívoco. Brota en mí - ¿de dónde?, ¿a través de qué?, no lo sé. Llega, independientemente de mi voluntad, habitualmente rodeado y oscurecido por una aglomeración de sentimientos, apetencias, aversiones, también de otros pensamientos, bastante a menudo apenas distinguible de un «querer»o un «sentir». Se lo saca de esa aglomeración, se lo limpia, se lo pone de pie, se mira cómo queda y cómo va, todo en un asombroso presto y, no obstante, sin el más mínimo sentimiento de la prisa: quién hace todo esto, - no lo sé, pero en todo este proceso soy sin duda más espectador que autor. [...] El origen del pensamiento permanece oculto; la probabilidad de que sea sólo el síntoma de una situación mucho más amplia es grande ${ }^{28}$.

26. FP III 556, 26[92].

27. Para una más amplia y exhaustiva explicación de la cuestión del pensamiento y de la conciencia me remito a L. Lupo, Le colombe dello scettico, Pisa: ETS, 2006 (sobre todo pp. 107 ss.). Sobre este tema véase también G. Abel, «Bewußtsein - Sprache - Natur. Nietzsches Philosophie des Geistes»: Nietzsche-Studien 30 (2001), 1-43, y C. J. Emden, Nietzsche on Language, Consciousness and the Body, Chicago: University of Illinois Press, 2005.

28. FP III 823, 1885, 38[1]. Puede ser útil gastar dos palabras para contextualizar este fragmento. Se encuentra en una sección de los Fragmentos póstumos muy interesante (grupo 38 de 1885), desde el momento en que en ella están presentes numerosas ideas luego reunidas en el grupo de parágrafos del 15 al 19 de Más allá del bien y del mal. En 38[3], por ejemplo, se encuentra una reanudación del fragmento relativo a la convicción de que el «yo» sea la causa de los pensamientos y de que pueda valer como certeza inmediata (este apunte es claramente una preparación de JBG \$ 16 , así como aquello que precede): «- - el yo lo pone el pensar; pero hasta ahora se creía, como cree el pueblo, que en el 'yo pienso' hay algo de certeza inmediata, y que este 'yo' es la causa dada del pensar, por cuya analogía nosotros entenderíamos todas las demás relaciones causales». $\underline{\text { ¿No es }}$ un caso? que de repente antes de eso haya un apunte sobre el pensar lógico, donde vuelve la idea de las ficciones regulativas, y que inmediatamente después se encuentre, al contrario, un pasaje que 
Como se puede ver, Nietzsche repite, ampliándolo, el mismo concepto, o sea, la idea de que el proceso que genera el pensamiento se desarrolla fuera de cualquier registro consciente, antes de la formación de una dimensión psicológica o absolutamente lógica. Llevando los procesos cognitivos a su fundamento fisiológico, Nietzsche observa en particular la falta absoluta de un sujeto que dirija conscientemente — voluntariamente- su desarrollo. Lo que sucede en el caso del pensamiento no es otra cosa que la necesidad de verificar hechos químicos y físicos, cuyos resultados llegan finalmente a nuestra conciencia. Nietzsche $\underline{\text { ¿se aleja retira? }}$ por lo tanto de cualquier forma de compromiso ontológico dirigido a responder a la pregunta relativa al agente de esta actividad. Su suspensión de juicio respecto a esto está sostenida por la idea de que se pueda ser simplemente espectadores del resultado de este proceso inconsciente y que la intervención del sujeto esté ligada solamente al aislamiento del pensamiento final de la masa de pulsiones de la que emana. El principal error de la lógica, que lleva a un mal entendimiento de los caracteres de la actividad del pensamiento y a la invención de un yo, consiste por tanto en esta atribución de un agente que guiaría la articulación de una dinámica absolutamente natural. En la práctica, Nietzsche apunta hacia una naturalización de los procesos del pensamiento, moviéndose de manera opuesta respecto a la práctica común de la espiritualización antropomórfica de la naturaleza.

El yo, privado del valor ontológico que la psicología tradicionalmente le atribuía y de su autonomía fuera de la cadena de sensaciones, revela su carácter de ficción lógica adoptada con el fin de ordenar el flujo de las percepciones ${ }^{29}$. Conforme a esta perspectiva de naturalización del pensamiento, Nietzsche lleva su propio razonamiento a consecuencias extremas, y pocos años después llega a escribir en su cuaderno un apunte en el que establece la hipótesis de la completa eliminación del dualismo entre sujeto y acto:

El «pensar», tal como lo suponen los teóricos del conocimiento, es algo que no sucede en modo alguno: eso es una ficción totalmente arbitraria, que se consigue al resaltar un único elemento del proceso y sustraerle todos los demás, un arreglo artificial con el fin de hacerlo comprensible...

El «espíritu», algo que piensa: a ser posible «el espíritu absoluto, puro, pur»esta concepción es una segunda consecuencia derivada de la falsa autoobservación que cree en el «pensar»: aquí se imagina en primer lugar un acto que no se produce, «el pensar», y en segundo lugar se imagina un substrato del sujeto en el que tiene su origen cada acto de este pensar y ninguna otra cosa más: es decir, tanto el hacer como el agente son ficticios ${ }^{30}$.

A lo que Nietzsche se refiere en este pasaje es naturalmente a una forma consciente de pensamiento, ese punto de llegada de una cadena de procesos que

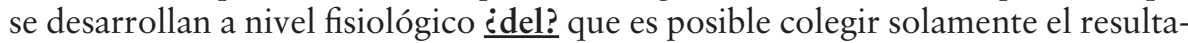
do último. Los dos agentes de esta relación son fruto de la traducción en un len-

contempla la «verdad» (nótese que JBG se cierra con la pregunta: «Pero ćpor qué entonces la verdad a toda costa?»). Y todavía, ojeando el cuaderno de 1885 , se puede leer un apunte sobre el tema de la «voluntad» que prepara JBG $\$ 19$, en el que volvemos a cuestiones correlativas al problema del sujeto como agente de las propias acciones.

29. Una conclusión que, como se ha visto, corresponde perfectamente a la perspectiva y a los términos de la de Mach.

30. FP IV 399, 1887-1888, 11[113]. 
guaje que es incomprensible para nosotros ¿̇de? una dinámica natural. Más aun, él vive en una dependencia recíproca del plano lógico y, tal como sucede para el «mundo verdadero» y para el «mundo aparente» de los que se habla en el Crepúsculo de los ídolos, la eliminación del primero determina la eliminación del segundo. En realidad no hay un "pensar», sino sólo la incesante articulación de fuerzas que se realzan a un nivel microscópico, de la misma manera que no hay una «mente», un sujeto identificable como «algo que piensa». Mente y pensamiento son definibles sólo uno a partir del otro; puesta de manifiesto la inconsistencia ontológica de la primera, el segundo también pierde significado. El dualismo es por lo tanto superado: más aún, completamente eliminado.

\section{MÁS ALLÁ DE LAS CERTEZAS INMEDIATAS}

¿Qué se puede concluir, entonces, de la relación mente-cuerpo tal y como es pensada por Nietzsche y Mach? Como se ha visto, desde el punto de vista ontológico toda distinción desaparece, revelándose una mera construcción artificial llevada a cabo por la lógica. El yo para ellos es un simple producto del pensamiento, una noción elaborada por la facultad cognoscitiva del hombre de acuerdo con aquel principio de economía que caracteriza éla actividad de él?. Describir los procesos mentales en términos de una relación entre sujeto y objeto es la afirmación impropia de un esquema de relación que no pertenece a ellos, pero que resulta esencial para los fines de la conservación de la especie, como material para orientarse en el mundo ${ }^{31}$. En la base de todo hay simples procesos fisiológicos, que son interpretados en un cierto modo en el momento en que sus efectos alcanzan el nivel consciente. De lo poco que se ha visto resulta por tanto claro cuánto insiste Nietzsche en querer reducir los estados mentales a sus componentes fisiológicos, a la corporeidad; aunque en realidad él no nos proporciona una definición particularmente clara de lo que entiende con el término «cuerpo", hecho en sí coherente con una posición contraria a toda forma de sustancialización metafísica que, en el caso en cuestión, llevaría a un desequilibrio descriptivo entre lo «físico» y lo "psíquico» ${ }^{32}$. En particular, en un fragmento de 1883, en la línea de las observaciones sobre el pensamiento que desarrollará en el periodo siguiente, Nietzsche llega a afirmar que «sólo hay estados CORPORALEs: los estados mentales

31. Véase en relación con esto lo que Nietzsche sostiene en FW $\mathbb{S}$ 110-111. Para un examen de la concepción biológica de la teoría del conocimiento de Mach nos remitimos a M. Čapek, «Ernst Mach's Biological Theory of Knowledge»: Synthese 18/2 (1968), 171-191.

32. Se puede encontrar también en esto una semejanza con la posición de Mach, cuyo objetivo programático es precisamente la superación de la distinción entre estados físicos y estados psíquicos. Sobre la cuestión de la falta de claridad en la definición del término «cuerpo» por parte de Nietzsche me sirvo de las observaciones de Luca Lupo: «La noción de cuerpo en la que piensa Nietzsche es problemática, no unívoca, no reducible a una forma de materialismo positivista ċen a una? forma de vitalismo: en lugar del término 'cuerpo', el filósofo utiliza la locución más cauta, 'lo que llamamos cuerpo', y de esta última palabra dice que es un 'símbolo' para indicar una actividad específica, es decir, la cooperación de una multiplicidad de seres. Nietzsche piensa en una noción de cuerpo como un campo de fuerzas, una entidad organizada plural y múltiple, en definitiva: un sistema de relaciones» (L. Lupo, Le colombe dello scettico, cit., p. 133). Sobre este punto véanse las observaciones de W. Gerhard, «The Body, the Self, and the Ego», en K. Ansell Pearson (ed.), A Companion to Nietzsche, Oxford: Blackwell, 2006, pp. 273-296. 
son sus consecuencias y símbolos» ${ }^{33}$. Además, en este apunte Nietzsche señala la necesidad de repensar el carácter metafísico propio del dualismo entre «estados internos» $\mathrm{y}$ «estados externos» $\mathrm{y}$, más en general, de toda forma de atribución de un ser, la cual se demuestra constitutivamente errónea, y entre las cuales se incluye la posición de un yo. El yo es por tanto un error, generado a partir de la interpretación del proceso fisiológico, del cual emergen las formas conscientes del pensamiento. Tal error se verifica de un modo muy específico, que Nietzsche describe en un apunte de 1885. En él el filósofo observa que la afirmación de un sujeto que sea causa del pensamiento es debido a un caso particular de falsificación del proceso natural, en el cual la causa de un pensamiento se cambia por el mismo efecto: si de hecho es el pensamiento el que produce el yo, es sin embargo este último el que es identificado como el verdadero agente.

El orden temporal invertido. El «mundo externo» actúa sobre nosotros: el efecto es telegrafiado al cerebro, allí es ajustado, configurado y reconducido a su causa: después la causa es proyectada y sólo entonces el factum nos llega a la CONCIENCIA. Es decir, el mundo de los fenómenos nos aparece sólo como causa después de que «él» haya actuado y el efecto haya sido elaborado. Es decir, nosotros invertimos continuamente el orden de lo que sucede. - Mientras «yo» veo, se ve ya otra cosa. Pasa como con el dolor ${ }^{34}$.

En este pasaje se aclara el punto de vista de Nietzsche, según el cual el pensamiento consciente es solamente el momento conclusivo de una larga cadena de acontecimientos desarrollados a nivel inconsciente y la afirmación de un sujeto como agente del pensamiento sucede por culpa de la incapacidad de ordenar de manera correcta la relación de causa y efecto. Con esto se vuelve al punto de partida, o sea, a la observación tomada de Lichtenberg presente en JBG 17: identificar un «yo» como condición del predicado "pienso» «es una falsificación del estado de los hechos». Más bien, se debería mantener el impersonal y corregir la «certeza inmediata» del cartesianismo con la idea de que el pensamiento llegue sin que exista un objeto externo que lo quiera.

Como se ha dicho, esta posición constituye un claro ejemplo de convergencia de las ideas de Nietzsche y Mach, y el fragmento en el que aparece representaría un lugar óptimo desde el que moverse para discutir la relación con él ${ }^{35}$. Sin

33. FP III 249, 1883, 9[41].

34. FP III 727, 1885, 34[54].

35. No tanto por la referencia a Lichtenberg, señal de ¿̇un trasfondo común de referencia?, sino por el tema específico de la inversión temporal que aparece también en un aforismo del Crepúsculo de los ídolos («Los cuatro grandes errores. Error de las causas imaginarias»). En 2003 Thomas Brobjer propuso la confrontación entre este último y una página de Análisis de las sensaciones de Mach, como testimonio de la influencia derivada de la lectura de esta obras ( $N$ Nachweis aus Mach, Ernst: Beiträge zur Analyse der Empfindungen»: Nietzsche-Studien 32 [2003], 450-451). En este caso tenemos el ejemplo de cómo la cronología no es favorable a una conexión directa de Nietzsche con Mach: de hecho, si bien los pasajes manifiestan correspondencias puntuales (los dos tratan del fenómeno onírico tomando como ejemplo ¿̨la registración? de un golpe de cañón y de una explosión), el tema principal del «vuelco temporal» en ellos examinado aparece más veces en los cuadernos de Nietzsche entre 1884 y 1885 , y por tanto en un periodo que precede a la publicación de la obra de Mach sobre las sensaciones. Además, en Humano demasiado humano se encuentra todavía presente, dentro de un parágrafo titulado "Lógica del sueño» (MA I, $\mathbb{1 3}$ ). En este aforismo se puede encontrar todos los elementos que vuelven a aparecer en el Crepúsculo de los ídolos, desde la cuestión 
necesidad de extenderse sobre cuestiones histórico-filológicas relativas a la reconstrucción de la relación entre estos dos autores, baste aquí con señalar que la convergencia de algunos temas de la filosofía de Nietzsche con las observaciones de un científico como Mach, cuyo nombre está ligado a la epistemología moderna y al desarrollo de la filosofía científica del siglo xix, es seguramente un dato significativo a tener en consideración para valorar su pensamiento. Por lo que se refiere al hecho de que sus observaciones probablemente hayan sido tomadas de otros autores, lo que está claro es el modo en que Nietzsche ha sabido reflexionar sobre tales cuestiones y cómo ha llegado ya a anticipar posiciones específicas que animarán el debate científico y filosófico en los años posteriores. En el caso de la psicología cognitiva, por ejemplo, sus ideas se enmarcan perfectamente en aquella línea de investigación que, pasando por encima del cartesianismo, se movía hacia una liberación de los residuos metafísicos y dualistas de la concepción de la relación cuerpo-mente. Sobre este punto son dignas de consideración las palabras pronunciadas por Daniel Dennett, el cual ha querido subrayar la capacidad de Nietzsche de introducirse en un debate que en muchos aspectos no debía ser el suyo. A su parecer, de hecho, «Nietzsche tenía una visión rica e importante -importante sobre todo para él, pero quizás también para nosotros- del fundamento materialista y evolutivo de la conciencia, tanto de lo humano como de lo biológico, que comportaban consideraciones no banales» ${ }^{36}$.

Esta posición refleja el intento antimetafísico más general ejemplificado por Mach, que por otra parte es el rasgo común de una época orientada hacia la denuncia del carácter ilusorio de las principales nociones con las que no sólo la ciencia y la filosofía, sino también el saber común han construido su descripción del mundo. La noción de yo constituye un objeto de particular relieve, precisamente por el modo en que convergen en él investigación científica y sentido común, y no es por lo tanto un caso que forme parte de aquellos ídolos eternos sobre los cuales Nietzsche considera que es necesario operar con intención crítica, con el fin de revelarnos su falta de contenido. La naturalización del pensamiento entra por lo tanto plenamente en aquella operación de liberación del yugo de la metafísica que es épuesta sobre el tapete? de modo particular en el Crepúsculo de los ídolos y con la cual Nietzsche trataba de abrir una nueva época para el pensamiento, creando las condiciones para el desarrollo de la filosofía del futuro de la que precisamente Más allá del bien y del mal había sido el preludio.

[Traducción: Luis Enrique de Santiago Guervós]

general de la inversión temporal al ejemplo específico del golpe de cañón adoptado en la argumentación. Para un análisis completo de la cuestión me remito a P. Gori, Il meccanicismo metafisico, cit., «Introduzione», $\mathbb{S} 3$.

36. D. Gambarara, “'Il pensiero umano non è tutto linguistico', entrevista a D. Dennett», $I l$ Manifesto, 23 de mayo de 2001. Esta cita se la debo a Luca Lupo, en su ya mencionado trabajo Le colombe dello scettico, p. $135 \mathrm{n}$. 